\title{
LA PLURIVOCIDAD DE 'BUENO'
}

AUGUSTO SALAZAR BONDY

Universidad de San Marcos, Lima

Ya Aristóteles observó que "el bien se toma en tantos sentidos como el ente" y que, por tanto, "no puede ser algo común, universal y único, pues si así fuese, no se predicaría en todas las categorías, sino en una sola." Como ha señalado a este propósito Jean $\mathrm{Wahl}^{2}$, se podría hablar de una analogía del valor paralela a la del ser. La observación de Aristóteles y el comentario de Wahl apuntan a un hecho patente de la experiencia lingüística de las palabras estimativas, cuyo ejemplar más caracterizado es 'bueno'. Se trata de la variación múltiple de sentido que manifiestan en el uso valorativo común. Queremos llamar la atención aquí sobre tres clases principales de variaciones de 'bueno' y proponer una explicación general de este hecho semántico.

\section{Clases de significado de 'bueno'}

Las variaciones a que nos referimos son las siguientes:

i] Con relación a cada género o especie de objetos - tomando 'objeto' en su acepción más general- se entiende 'bueno' en sentido diferente en la medida en que tiene que ver con determinadas propiedades, distintas en cada caso. Cuando se habla de un buen automóvil se comunica algo diferente que cuando se habla de un buen libro. Hay pues lo bueno (o el bien) de cada objeto.

${ }^{1}$ Etica Nicomaquea, trad. Gómez Robledo, Universidad Nacional Autónoma, México, 1954, I, VI, p. 119.

${ }^{2}$ Introducción a la filosofia, Fondo de Cultura Económica, México, 1950, p. 294. 
ii] Algunas veces se habla de algo bueno en sentido absoluto y se lo opone a lo bueno relativo. Dentro de la mis. ma linea de variaciones semánticas se encuentran otras pa. rejas de conceptos, a saber: bueno intrinseco y extrinseco, bueno como fin y como medio, bueno primitivo y derivado (o irradiado, trasferido, etc.). Es preciso considerar aquí también las diferencias correspondientes a los juicios valorativos de comparación y de grado.

iii] Hay las diferencias correspondientes a los diversos órdenes valorativos: estético, moral, económico, religioso, vital, etc. 'Bueno' puede entenderse, pues, unas veces en sentido moral, otras en sentido estético, y asi sucesivamente.

${ }_{i}$ No conspira esta multiplicidad de sentidos - por partida triple en nuestra enumeración anterior - contra la unidad semántica de 'bueno'? Cuando con respecto a un término ordinario encontramos una tal variedad de sentidos hablamos, por ejemplo, de homonimia o de traslación, alteración o pérdida de sentido. En estos casos, por lo general, las palabras ven comprometida su unidad semántica con tal comportamiento. El caso de 'bueno' es muy diferente. Lejos de afectar la unidad de su uso y función, la plurivocidad parece constituir un rasgo esencial del sentido y abrir el camino para la comprensión de la peculiar naturaleza lingüística de la palabra.

Veámos sobre qué bases se ofrece esta unidad de comportamiento semántico del término 'bueno'. Está dada por vivencias que presentan la misma estructura esencial en todos los casos. Se trata de comportamientos de adhesión o rechazo referidos a un determinado elemento o complejo objetivo. En este punto contrasta marcadamente una vivencia cognoscitiva referente a objetos físicos, animados, naturales o artificiales, con una vivencia enderezada a algo bueno, sea esto mentado como bueno absoluto, relativo, final o instrumental, sea como estético, moral, religioso, etc. Quien pronuncia u oye la palabra 'bueno' se comporta con respecto al objeto, básicamente, de la misma manera, es decir, con una intención favorable o pro, que contrasta con lo que su- 
cede en el caso de una palabra neutra del tipo de 'piedra', 'triángulo', etc. ${ }^{3}$

No obstante esto, existen las diferencias señaladas, tanto entre las clases de variaciones cuanto entre los diversos usos de 'bueno' dentro de cada clase. Encontramos, pues, contraste y unidad, lo cual no puede explicarse sino pensando en un tipo especial de palabra, capaz de vincularse con contenidos ónticos varios, de absorberlos y asumirlos en cierto modo, sin quedar, empero, fijada en su sentido por tales contenidos. Este tipo especial de palabras puede caracterizarse - en una primera aproximación- como términos formales. Quiere esto decir que, como tales, estos términos no comunican ningún contenido óntico, pero pueden adecuarse a diversos contenidos y especificar con ellos su significación, al tiempo que califican los complejos objetivos correspondientes.

Es ilustrativo comparar a este respecto el comportamiento de adjetivos como 'azul' o 'izquierdo' con el comportamiento de 'bueno'. En el primer caso hay un elemento concreto del objeto y sólo uno, ese determinado color que él tiene, consignado en el sentido de la palabra. Ésta comporta un momento semántico que afecta al objeto. El objeto es azul, o sea que posee una propiedad que no es contextual y variable, en el sentido en que lo es, por ejemplo, una relación, sino intrínseca y fija. Por otra parte, en el sentido de 'azul' no interviene para nada el resto de las determina. ciones constitutivas del objeto, de tal manera que se puede entender 'azul' sin pensar en ningún objeto azul particular. En cambio, 'bueno' es, por un lado, una palabra que varía de sentido en cada caso, incorporando las determinaciones de los objetos - de allí que no se pueda saber qué quiere decir 'bueno' hasta que no se sepa si se está hablando de automóviles, manzanas o libros-y, por otro lado, no queda fijada en su sentido por este comercio con los contenidos, pues cuando se oye hablar de buenos automóviles, pri-

3 Se entiende que no consideramos el caso de un uso de estas palabras con intención valorativa, que es también posible. 
mero, y luego de buenos libros, no se deja de entender también algo invariable en ambos casos. Justamente, si la palabra comunicara sólo ese elemento variable se diversificaría en una pluralidad de términos sin conexión mutua o, en todo caso, no ligados de la manera en que se dan de hecho en nuestra experiencia del lenguaje ordinario.

Por lo que toca a 'izquierdo', es un término abstracto y relativo. Es abstracto en cuanto no incorpora nunca en su significación ningún momento singular y constitutivo de los objetos a los que se aplica; relativo, en tanto su sentido indica una posición recíproca de dos o más objetos. Cuando decimos de, por ejemplo, un árbol, que es el izquierdo, no mentamos nada que lo constituya como propiedad; marcamos tan sólo una situación relativa a otros árboles u objetos y al sujeto que habla. Por otro lado, aplicado a árbol, 'izquierdo' no gana ningún elemento nuevo, concreto, es decir, no queda especificado, como tampoco ocurre en su aplicación a cualquier otro objeto. Comparándolo con 'bueno', observamos que se asemeja a éste en la posibilidad de predicarlo de muchos objetos, diciendo asi de ellos algo constante, pero se diferencia de él en que, de una parte, lo que se dice es una relación (o sea, un elemento descriptivo, "natural" o fáctico) y, de otra, no incorpora ningún elemento intrínseco o constitutivo del objeto, ninguna de las notas propias que lo singularizan, como ocurre con 'bueno'.

Esquemáticamente y sólo como un expediente de aclaración podría afirmarse que la significación de 'azul' apunta a lo intrínseco del objeto y la significación de 'izquierdo' a algo extrínseco al objeto, mientras que la de 'bueno' participa de ambos momentos, sin mentar propiamente ni lo intrínseco ni lo extrínseco del objeto 4 .

A este respecto es ilustrativo llamar la atención, como lo hace Urmson ${ }^{5}$, sobre el hecho de que no se puede aprender

4 Con este hecho se vincula la conocida paradoja de Moore. Cf. "The Conception of Intrinsic Value", Philosophical Studies, Routledge and KeganPaul, London, 1922, p. 273.

s Cf. J. O. Urmson, "On Grading", in Logic and Language, 2d. Series, A. Flew ed., Basil Blackwell, Oxford, 1961. 
a usar el término 'bueno' de la misma manera como se aprende el uso de otras palabras. Consideremos, por ejemplo, el caso de 'azul'. Es posible pasar del empleo de esta palabra, aplicada v.g. a trajes, a su uso tocante a un género distinto de objetos, como los automóviles, los libros, etc. Hay un solo uso correcto de 'azul', que se aprende de una vez por todas. Esto no ocurre con 'bueno', lo cual refuerza la opinión de que, mientras 'azul' comunica un contenido óntico determinado, 'bueno' se comporta de diversa manera. Lo mismo puede decirse de 'izquierdo', que tampoco presenta dificultades de aplicación una vez aprendido.

Pasemos ahora a considerar la conexión entre lo invariable y las variaciones del sentido de 'bueno' desde un punto de vista general. Como hipótesis semántica capaz de cubrir todos los casos, proponemos la siguiente: 'bueno' comunica una exigencia de actitud favorable referida a un elemento o complejo objetivo determinado ${ }^{6}$, que se incorpora al sentido de la palabra en cada uso particular. La actitud y el complejo objetivo varían según los casos, en cambio la exigencia permanece inalterada y comporta un momento de imperatividad universal e incondicionada, de orden no fáctico. Por ser constante la exigencia y por tratarse sólo de una demanda o necesidad no fáctica - como es la necesidad lógica, aunque ésta no se identifique con la axiológica-, 'bueno' tiene un sentido unitario y no comunica ningún contenido óntico. Pero, puesto que los elementos y complejos objetivos mencionados varían según los casos, tanto en su naturaleza cuanto en sus conexiones situacionales y en sus funciones, y puesto que el carácter y el grado de las actitudes favorables varían de acuerdo con la múltiple gama de los comportamientos humanos (afectivos, voluntarios, intelectuales, imaginativos, conscientes, extraconscientes, etc.), se explica que la palabra de que tratamos se ofrezca como plural sin resultar afectada, no obstante, en su unidad fundamental. Si esto es así, se comprende mejor asimismo que el

6 Como antes, tomamos aquí 'objetivo' en el sentido más amplio, que cubre todo término intencional o sujeto de un juicio. 
aprendizaje de 'bueno' difiera del de otras palabras - co. mo, v.g., las antes examinadas: 'azul', 'izquierdo'. Hay algo de común que se comunica siempre al usar 'bueno', pero como esto no es un hecho sino una exigencia, como no es un contenido óntico sino un imperativo o una forzosidad, es imposible saber de una vez por todas qué es lo exigido, a qué objeto o a qué características debemos adherir. Esto hay que aprenderlo en cada caso, aunque en todos se mantenga la demanda de actitud favorable.

A partir de este planteo básico, vamos a intentar explicar, en un segundo nivel de análisis, las diferencias corres. pondientes a cada tipo y caso de variación ${ }^{7}$. Al hacerlo, tendremos en cuenta los factores del fenómeno evaluativo que, como hemos visto, se reflejan en la hipótesis semántica general propuesta. Estos factores son: a) el objeto (elemento o complejo objetivo); b) la vivencia (favorable o desfavorable); c) la exigencia de actitud (o imperatividad o forzosidad). Echando mano de estos factores y apelando a variaciones $\mathrm{y}$ funciones de unos $\mathrm{u}$ otros $\mathrm{o}$ a sus relaciones, según los casos, creemos que puede darse cuenta satisfactoriamente de las diferencias que nos interesan.

\section{El valor propio de cada género de objeto}

Como ha señalado muy justamente $\mathrm{Hare}^{\mathrm{s}}$, el sentido evaluativo de palabras como 'bueno' está ligado a un contenido descriptivo que comunica de ordinario la misma palabra. Aunque no se identifique con él, está fundado en él. Nosotros creemos que es necesario enfatizar - como asimismo lo ha hecho Hare- la relación y la diferencia que existe en las palabras evaluativas entre el contenido descriptivo y el momento propiamente valorativo, esto es, el hecho de que, en cada caso, 'bueno' por ejemplo, tiene que ver con determinadas propiedades del objeto, pero no las menciona

T Lo cual, por lo demás, servirá asimismo como medio de control de nuestra hipótesis.

s. Cf. The Language of Morals, Oxford University Press, 1952. 
simplemente, como ocurre con un término descriptivo. Cuando se dice, v.g., que un automóvil es bueno, se comunica un juicio de valor que implica el decir que el automóvil tiene tales y cuales propiedades, a condición de que dichas propiedades estén consignadas en una norma de bondad, o sea, a condición de que ellas figuren no como elementos ónticos que se describen, sino a manera de signos de una condición especial del objeto, la condición de valioso.

Esta función especial que cumple el contenido descriptivo de 'bueno' y la imposibilidad de reducir a él el sentido evaluativo de esta palabra se ven claramente en el caso de aquellos términos que, cumpliendo ordinariamente una función preeminentemente informativa, han pasado a desempeñar también una función evaluativa, tanto en su uso propio como es su uso metafórico o traslaticio. Tomemos, por ejemplo, la expresión 'de acero', cuyo sentido y uso ordinarios son informativos. Decimos que una espada es de bronce, de hierro o de acero, en plan descriptivo normal y usando las palabras en su sentido propio. Pero como el acero es un material muy apreciado por su dureza y elasticidad, 'de acero' ha pasado a usarse en sentido evaluativo, de tal modo que al decir que una espada es de acero queremos comunicar también una condición de valor. Se observa aquí la íntima unión del contenido informativo y la función valorativa, así como la importancia que tiene el momento estimativo para la fijación de las notas descriptivas de los términos de valor, es decir, para la definición del objeto apreciado. En efecto, en el pasado, cuando se quería comunicar la condición de valiosa de una espada, no se decía 'de acero' sino 'de bronce', habiendo caído luego en desuso esa expresión. Inversamente, se observa aquí asimismo la importancia de la experiencia de tal o cual propiedad o hecho en la configuración del sentido estimativo variable de una palabra, pues el comportamiento del material de hierro, bronce o acero, en la fabricación de armas, cuenta decisivamente para la estipulación del uso encomiástico del término 'de acero'. De otro lado, la diferencia 
entre una expresión valorativa y un término simplemente descriptivo, esto es, la irreductibilidad del segundo al primero se ve claramente en el hecho de que es posible decir del acero que es bueno o excelente, sin que el enunciado sea necesariamente analítico. Esto significa que el sentido informativo del término 'de acero' no se identifica con el sentido evaluativo de 'bueno'. Más bien se articula con él como resultado de una vivencia evaluativa dirigida al acero y sus propiedades.

Resumiendo lo anterior, puede decirse que, en razón de una apreciación dirigida a las propiedades del acero en lo que toca a la fabricación de espadas, el término 'bueno' (o 'excelente') resulta igual a 'hierro, más carbono, niquel, vanadio, en determinadas proporciones, que ofrece una dureza y una elasticidad determinadas', que, a su vez, es igual a 'de acero'. En otro contexto, 'excelente' o 'bueno' será igual a otras propiedades. Pero, en ningún caso, 'de acero' y 'hierro, más carbono, etc.', serán, sin más, por naturaleza diriamos, iguales a 'excelente'.

Según indicamos, una vez sabido qué quiere decir 'bueno' aplicado a autos o espadas, no se sabe todavía qué quiere decir aplicado a otros objetos. $\mathrm{O}$ sea, una vez que se sabe cuáles y cómo son los objetos buenos de un determinado género, no se sabe aún cuáles y cómo son los objetos buenos de otro género. Por tanto, 'bueno' significa en un caso algo distinto de lo que significa en el otro. De cada género de objetos y, en el extremo límite, de cada objeto, se predica 'bueno' en un sentido que comporta diferencias, a pesar de la unidad semántica básica de la palabra.

¿De dónde proviene la diferencia en este caso y cómo se concilia con la semejanza en el uso general? La diferencia, creemos, puede explicarse por la variación de los contenidos objetivos (propiedades, relaciones, estados, etc.) mentados en cada caso, que se refieren a los varios géneros de objeto apreciados. Hay un complejo objetivo propio del automóvil - según los standards actuales-, el cual se incorpora al concepto de bueno cuando éste es aplicado hoy 
día a los autos. Otro complejo diferente - susceptible de ser, en principio, completamente diferente- corresponde a las espadas y otro a las mesas, los cuales se incluirán en los respectivos conceptos de bueno aplicados a dicho género de objetos?.

De otro lado, hay un elemento constante, comunicado en todos estos casos. Cuando se habla de un automóvil bueno, una espada buena o una mesa buena, se dice algo semejante en los tres casos, y es, a saber, que 'debe tenerse res. pecto a tales objetos una actitud favorable'. Lo común que comunica 'bueno' es, pues, una exigencia de actitud positiva referida ora a este complejo objetivo, ora a aquel, con lo cual la diversidad se concilia con la unidad del término.

\section{Valor absoluto y valor relativo. Grados de valor}

Son formas corrientes del lenguaje estimativo las que ilustran frases como 'El amor es intrínsecamente bueno', 'La felicidad es buena como fin último', 'Este libro es bueno para él', 'En relación con su estado de ayer el enfermo está bien hoy', 'Juan es mejor que Pedro', 'La nota 20 es la óptima'. En ellas, la palabra 'bueno' y sus derivadas o afines desempeñan, a la vez, un papel común y una función de sentido diferente en cada caso. Se trata, en primer lugar, de la diferencia entre las predicaciones de bueno en términos absolutos y en términos relativos, que se conecta con el problema, largamente discutido en axiología, de los valores absolutos y relativos, extrínsecos e intrínsecos, fines y medios, etc. Con esta diferencia se vincula asimismo el contraste que se establece en los juicios de valor cuando se comparan y gradúan los objetos juzgados. Hay pues aquí una nueva clase de variantes de 'bueno' que debe ser explicada en su diversidad y en su articulación con la unidad semántica de esta y otras palabras estimativas.

Veámos en primer lugar el caso de los llamados valores absolutos, es decir, de aquellas predicaciones que se ofre-

\footnotetext{
0 Repárese en que esta variación puede referirse al mismo objeto, como ocurre con los cambios de estilo o moda.
} 
cen como atribuciones irrestrictas de valor. Esto ocurre, v.g., en el uso de expresiones como "intrínsecamente bueno' o 'bueno en si'.

Cuando de un objeto se dice que es intrinsecamente bue. no, sólo se tiene en cuenta, al apreciarlo y al formular el juicio de valor respectivo, sus propiedades intrínsecas, o sea, aquellas que lo constituyen como tal, con prescindencia de los demás objetos, de las relaciones que lo vinculan con éstos, de su ambiente y, en general, de todo complejo situacional. La diferencia entre propiedades constitutivas y externas, es decir, entre lo intrínseco y lo extrínseco del objeto, es por cierto relativa y su determinación está sujeta a criterios variables. Por tanto, su uso normal se sitúa dentro de determinados límites de aproximación. Pero esto no afecta al análisis que queremos presentar. Su validez se mantiene, en efecto, aunque cambie en cada caso lo que se entiende por extrínseco e intrínseco respecto a un objeto. Dicho de otro modo, si cabe hablar de lo intrínseco y propio de un objeto, entonces tiene sentido hablar de algo intrinsecamente bueno. El significado de este 'bueno intrínseco' es lo que se trata de definir.

Sostenemos que cuando de un objeto se dice que es intrínsecamente bueno, el núcleo estimativo de la palabra 'bueno' - articulada como está, normalmente, con otros elementos semánticos descriptivos, expresivos y operativoscomunica una exigencia incondicionada de actitud pro o favorable respecto a él, con indiferencia o exclusión de las demás instancias existentes. Que algo sea intrínsecamente bueno significa entonces que hay que tener respecto a él, sean cuales fueran las circunstancias que lo rodean, una actitud favorable.

Afirmar que un objeto es valioso por si mismo o esencialmente bueno o que posee un valor propio o interno, son variantes del mismo género de predicación. A nuestro juicio, pueden ser analizadas de la misma manera: 'Debe tenerse hacia el objeto por sí mismo (o en su esencia, o en lo que le es propio o interno) una actitud pro o favorable'. 
El concepto de intrínsecamente bueno está estrechamente vinculado a otro concepto axiológico, el de valor final o valor-fin, que puede ser definido de acuerdo a la misma pauta analítica. En efecto, al decir de algo que es bueno como fin se comunica la exigencia de tener una actitud favorable a dicha cosa, tomada como fin en sí, esto es, tomada como algo que no está ordenado a la consecución de otra cosa, sino que se ofrece como término último de una serie de realizaciones.

Pasemos ahora a considerar el otro tipo de enunciados valorativos mencionados al principio, aquellos que, en contraste con las atribuciones de valor intrínseco o final, hablan justamente de lo valioso de un objeto como relativo o condicionado. Se trata de frases en que el miembro principal es el constituido por las palabras 'bueno para' o 'bueno en relación con'.

Pueden distinguirse a este respecto varios casos, ilustrables por sendas frases. Señalemos, en primer lugar, la existencia de un tipo de enunciados con las palabras indicadas, pero que, a primera vista, parecen no corresponder al género que queremos examinar aquí, sino, en parte por lo menos, al que hemos estudiado antes. Cuando v.g., se dice que algo es (o está) bueno para depósito, se hace referencia explícita a un objeto en su estructura, función o condición propia; tenemos por tanto una frase que contiene también una atribución absoluta de valor. Pero hay aquí, embozada las más de las veces, una valoración relativa. Esto se hace perceptible - con un matiz despectivo especial- en frases negativas del género de 'No está mal para...'; por ejemplo: 'El cuadro no está mal para (ser de) un amateur'.

Más claro es el caso de las dos siguientes oraciones: 1) 'El castigo es bueno para lograr un comportamiento correcto', y 2) 'Esta película es buena para mí. En la primera, tras 'para' tenemos una relación de medio a fin, por la cual un objeto o hecho es valorado en tanto que se ofrece como una instancia ordenada a la realización de un fin, y es va- 
lorado sólo en esta conexión. Tenemos, pues, aquí un caso claramente opuesto al de lo intrínsecamente bueno y también al de lo bueno como fin. Sin embargo, el análisis en términos de debe no requiere ser variado sustancialmente, pues se juzga al objeto en su conexión con algo exterior a él. En esta conexión objetiva, de medio a fin, que lo define como tal, es calificado de bueno, es decir, es tomado como algo respecto a lo cual hay que tener una actitud favorable (en la mencionada conexión). La exigencia del debe sigue siendo tan plena e incondicional como en el caso de lo intrínsecamente bueno, aunque el objeto mismo no sea considerado incondicionalmente sino, justamente, en una relación determinada.

En lo que toca al segundo caso, el de frases del tipo de 'Esta película es buena para mi', señalemos por lo pronto una doble significación, que puede pasar desapercibida, confundiendo el análisis. En enunciados de este tipo se pueden dar a entender, en efecto, dos cosas: $a$ ) que el objeto es valioso a juicio de un sujeto (el que habla, en este caso); b) que se adecúa al modo de ser de dicho sujeto y que esa adecuación es positiva desde el punto de vista del valor. La diferencia entre ambos sentidos se hace más clara si se advierte que en el primer caso es usual anteponer el 'para mi' ('Para mi es buena esta película'). La existencia de frases compuestas como la siguiente: 'Este medicamento es bueno para Julio, aunque él no lo juzgue así', en donde la afirmativa de una predicación no contradice la negativa de la otra, hace también patente la mencionada diferencia. Confirmando esto, el análisis de ambos enunciados es di. verso, aunque se siga sin dificultad el mismo principio de definición ya establecido. La frase "Esta película es buena para mí, entendida en el primer sentido, se analizará de la siguente manera: 'Debe tenerse ante esta película una actitud favorable, a juicio mio. ${ }^{10}$ En cambio, entendida en el

10 Sobre la función de la frase 'a juicio mio' son pertinentes las observaciones de Urmson a propósito de los verbos entre paréntesis. Cf. "Parenthical Verbs", Mind, Oct. 1950. 
segundo sentido, se analizará en estos términos: 'Debe tenerse una actitud favorable hacia esta película en cuanto adecuada a mí (personalidad, carácter, modo de ser, etc.)'. Como se observa, pese a disparidades notorias, no hay necesidad de modificar en nada la traducción de 'bueno' en términos de una exigencia de actitud favorable.

$\mathrm{La}$ segunda de las variantes que acabamos de analizar nos remite a aquellas frases en que la valoración tiene que ver con una relación objetiva o con una comparación explicitamente mentada. Así ocurre con los enunciados del tipo de 'Esta máquina es buena al lado de aquella, pero mala frente a la de más allá'. Se trata de juicios valorativos de comparación que son sólitos en los actos de preferencia, elección y establecimiento de órdenes jerárquicos. En este caso, el uso de bueno relativo se emparenta con el uso de los comparativos y superlativos ('mejor', 'peor', 'óptimo', 'pésimo'), todos los cuales poseen como núcleo semántico un ingrediente intencional de bueno en relación $a . .$.

Creemos que tampoco en este caso hay necesidad de variar el análisis propuesto para dar cuenta de los enunciados de valor relativo. La razón principal es que 'bueno en relación a' comunica una exigencia de actitud favorable a una cosa contrastada con otra, y equivale, en última instancia, a 'mejor', cuyo análisis puede hacerse en términos de exigencia de preferir o de ser favorable a $X$ antes que a $Y$. Decir, en efecto, que estos zapatos comparados con aquellos son buenos, equivale a decir que son mejores, lo cual significa, en nuestro modo de análisis, que debe tener. se una actitud favorable con respecto a los primeros antes que (o más bien que) a los segundos, o que debe preferirse los primeros a los segundos.

Hemos mencionado antes, al lado de 'mejor' y 'peor', los superlativos del tipo de 'óptimo' y 'pésimo'. Estos y otros términos significativos de grado se vinculan indudablemen. te con los que acabamos de analizar. Pero, justamente en la medida en que comunican grados de valor, presentan un problema de interpretación que es conveniente encarar 
por vía de ratificación y matización de lo que hemos dicho hasta aquí.

Cuando alguien afirma que $X$ es bueno dice que hay que tener respecto de $\mathrm{X}$ una actitud favorable. Si dice que $X$ es mejor que $Y$, significa que hay que tener una actitud favorable con respecto a $\mathrm{X}$ antes (o más bien) que con respecto a $Y$. En ambos casos se trata de una misma exigencia de actitud favorable; pero, en el segundo, la actitud favorable está referida a un objeto en contraste con otro y supone por tanto que se da como un acto de preferencia. En cambio, cuando se dice que $X$ es muy bueno o que es óptimo, se introduce un elemento de grado. Tal frase se diferencia de las otras frases no en la referencia a un término o a dos sino en este elemento de grado. Qué significa esta diferencia de grado y cómo se comporta con respecto al problema de lo absoluto y lo relativo del valor es una cuestión que puede servir mucho para aclarar el sentido de las palabras valorativas y el alcance de su uso. Vale la pena, por tanto, tratarla siquiera brevemente.

Digamos, en primer lugar, que hay una doble posibilidad de interpretar la graduación que aquí nos interesa: puede ponerse en la cuenta 1] del 'debe' incorporado al definiens o, 2] de la actitud favorable, que es el otro elemento decisivo de la definición. De acuerdo al primer proceder, el uso de 'óptimo' o de 'muy bueno' comunica un grado (sumo) de la exigencia de actitud favorable. En el segundo caso, ese uso comunica un grado (sumo) en la positividad de la vivencia, en la proyección favorable al objeto; en otras palabras, la frase dirá que hay una actitud muy favorable o favorable en grado sumo, a un objeto.

Estas dos posibilidades interpretativas, que se presentan como satisfactorias ambas dentro de ciertos límites, no son equivalentes. No cabe, en efecto, reducir un análisis en términos de grado de la exigencia, es decir, de grados del debe, a un análisis en términos de grados de actitud favorable. Observemos, por otra parte, que la primera de las interpretaciones parecería ser más propia de la línea gene- 
ral de análisis aqui defendida, pues la palabra 'debe' (o sus equivalentes), con su exigencia característica, es fundamental para descartar toda posible reducción naturalista. Sin embargo, nosotros nos inclinamos más bien por la segunda definición, por una razón fundamental, que es la necesidad de entender el 'debe' del definiens en un sentido distinto a cualquier mandato psicológico $\mathrm{y}$, por tanto, ajeno a las variaciones o cambios de intensidad propios de las vivencias. Habría el peligro de psicologizar la exigencia es. timativa si se admite una mayor o menor solicitación en lo debido, un debe que se impone con más leve o más fuerte exigencia. En cambio, esta variación puede perfectamente aceptarse de la actitud favorable, por ser una vivencia cuya propensión o rechazo con respecto al objeto puede variar en intensidad y fuerza, al tiempo que se mantiene constante la exigencia. Según esto, decir que $X$ es óptimo es decir que hay que tener con respecto a $\mathrm{X}$ el máximo grado de propensión o la actitud más favorable.

Una consecuencia importante de lo anterior es que el debe, la exigencia de actitud favorable, se ofrece como incondicional no sólo en el caso de los llamados juicios de valor absoluto sino también en los juicios en que se ha. bla de un bueno relativo o de un grado de valor. Decir (estimativamente) que algo es bueno significa siempre, pues, comunicar un momento de necesidad, de imperiosidad, sean cuales fueren las variantes de la predicación y los objetos a los cuales se refiere el juicio. Trasladado al lenguaje ordinario, con la terminología hipostasiante que es usual en él, esto significa que lo absoluto y lo relativo no corres. ponden propiamente a los valores sino al modo como son dados o tomados los objetos valorados. Dicho respecto a ellos, 'bueno' comunica siempre, en su núcleo estimativo, un requerimiento incondicional.

La explicación anterior, si es satisfactoria, da cuenta de las diferencias de sentido y uso que ofrece 'bueno' cuando aparece en predicaciones absolutas o relativas y graduadas. Como se ha visto, en el primer caso, la variedad proviene 
de la manera como se toma al objeto (por sí sólo o en relación con otros, como fin o como medio, etc.) y, en el segundo, de la mayor o menor intensidad, del grado de la adhesión o el rechazo que presenta la vivencia apreciativa. Pero ni las diversidades tocantes al objeto ni las correspondientes a la vivencia afectan la unidad y el carácter de la exigencia, que es el momento nuclear del sentido valorativo. Porque todas las palabras valorativas comunican una imperiosidad de adhesión o rechazo, sean cuales fueren los objetos y los estados psicológicos implicados, por eso también aquí hay unidad en la diversidad semántica del lenguaje del valor.

\section{Los diversos órdenes de valor}

$\mathrm{Si}$ examinamos las siguientes proposiciones: 'Esta novela es buena', 'Esta acción es buena', 'Esta máquina es buena', 'Esta penitencia es buena' y 'Esta manzana es buena', com. probaremos que en ellas aparece el término 'bueno' cum. pliendo la misma función y que, sin embargo, en cada caso se trata de un modo diferente de decir que algo es bueno. Estos modos corresponden a lo que vamos a llamar órdenes de valor. En efecto, cuando hablamos de una novela en términos encomiásticos, se afirma de ella que es buena esté. ticamente; cuando aprobamos una acción, esto tiene fre. cuentemente el sentido de una calificación moral; cuando decimos que una máquina es buena implicamos que lo es económicamente; mientras que, por lo general, una peniten. cia es buena religiosamente y una manzana se califica de tal desde el punto de vista hedonístico o vital. Con ello es. tamos poniendo de resalto la existencia de tipos de califica. ción estimativa a los cuales corresponden sendos sentidos de 'bueno'. Algunos de los principales entre estos sentidos son el estético, el moral, el jurídico-social, el económico, el religioso, el hedonístico-vital ${ }^{11}$, el teórico. Examinémoslos

\footnotetext{
11 Por comodidad y en razón de que no nos interesa entrar aquí en las dis. tinciones más finas, tomaremos en conjunto el elemento evaluativo hedonístico
} 
de cerca, a fin de ver en dónde reside el elemento diferencial que se articula con la unidad semántica básica de 'bueno', para lo cual tomaremos en cuenta, una vez más, los elementos que comporta el fenómeno estimativo.

a] Lo bueno estético. La experiencia que ofrece la base para una calificación valorativa estética es una vivencia en que predominan los momentos imaginativos, esto es, una vivencia de la fantasía, sea pura, sea vinculada a elementos perceptivos o intelectuales. 'Bueno' en su uso estético tiene que ver con tal tipo de comportamiento psicológico. Lo que comunica como exigencia es la demanda de una actitud de este tipo que, además, sea favorable. Haciendo jue. go con este factor vivencial, el objeto se presenta también con caracteres específicos. Un objeto no es estético sino en tanto que es considerado en su puro aparecer o como mera posibilidad irreal. Los factores de efectividad - no los de concreción sensible - quedan fuera del marco objetivo pertinente. Por tanto, decir que $X$ es bueno (estéticamente) es afirmar que debe tenerse ante la pura apariencia o la posi. bilidad irreal $X$, dada en una vivencia esencialmente imaginativa, una actitud favorable.

Lo dicho no impide reconocer posibles diferencias, tanto del lado vivencial como del lado objetivo, correspondientes a los casos de objetos estéticos naturales y artísticos, inanimados, animados y humanos, $\mathrm{o}$ a las especies más circunscritas de entidades artísticas (lo trágico, lo cómico, lo elegante, etc.). En todos estos casos, sin embargo, no deja de valer la interpretación general de lo bueno estético que he. mos propuesto.

b] Lo bueno moral. Las experiencias morales se caracterizan por dos notas esenciales: son voluntarias y compor$\tan$ un elemento ineliminable de racionalidad. Decir que algo es bueno desde el punto de vista moral es formular un juicio en que interviene, siquiera episódicamente, un

y el vital, asi como el social y el jurídico, que frecuentemente se separan al estudiar las clases de valor. Con ello no afectamos, sin embargo, la naturaleza de estos valores, que tienen mucho de común. 
momento de reflexión intelectual, y que se vincula, directa o indirectamente, con una decisión de la voluntad. Otro rasgo más debe ser consignado en esta descripción: aquello de lo cual se habla, el sujeto de la predicación, es siempre una acción o la unidad personal de un conjunto o proceso de acciones. Moralmente bueno es sólo un acto querido, una decisión tomada, una omisión voluntaria o una persona en tanto que quiere, hace u omite algo deliberadamente. De acuerdo con esto, los enunciados valorativos morales comunican una exigencia referente a vivencias reflexivas y voluntarias y se refieren a acciones que, en principio, tienen asimismo carácter reflexivo y voluntario.

Según lo anterior, decir que $X$ es (moralmente) bueno significa afirmar que debe tenerse respecto a una acción voluntaria $\longrightarrow 0$ a la unidad estructural de ellas que es una persona- una actitud reflexiva y voluntaria favorable. Con lo cual se echa de ver el carácter específico de la imperatividad moral, que compromete la decisión voluntaria de quien juzga buena una conducta. Esto es lo que se quiere comunicar cuando se habla del carácter obligatorio de la moralidad. Si alguien dice que respetar a su prójimo es (moralmente) bueno, está reconociendo una exigencia de actuar así y formulando una obligación que le concierne como sujeto moral, con respecto no a acciones meramente posibles sino a actos efectivos, dependientes de su arbitrio.

c] Lo bueno económico. En las valoraciones económicas predomina el momento intelectual, sin perjuicio de los factores afectivos que siempre acompañan a las vivencias de interés. Apreciar económicamente algo comporta normalmente un cálculo realista, una determinación de medios y fines, de causas y efectos, que pide la intervención de las funciones psíquicas racionales. A este predominio del intelecto corresponde, del lado del objeto, la presencia de una capacidad efectiva de intervenir en el proceso de la producción, combinada con un factor esencial de ahorro de esfuezo. Hay pues un realismo y un causalismo inherentes al 
fenómeno valorativo económico, tanto en su vertiente vivencial cuanto en su vertiente objetiva.

Según lo anterior, una frase estimativa del tipo de $X$ es (económicamente) bueno se traducirá en una exigencia, fundada en una operación intelectual más o menos explícita y compleja, de tener una actitud favorable a un conjunto objetivo determinado, de acuerdo a su capacidad de producir bienes y, al mismo tiempo, de ahorrar trabajo. Una traducción posible del sentido comunicado por $X$ es bueno (económicamente) es: 'Debe tenerse frente $a X$, de acuerdo a su capacidad de servir para la obtención del máximo de bienes con el mínimo de esfuerzo, una actitud positiva'.

Se comprende que este sentido resulte matizado según se juzguen los efectos mediatos o inmediatos, a corto o largo plazo, del hecho valorado, y según se tomen en cuenta otras variaciones, como son la mayor o menor capacidad de producción en relación con el mayor o menor ahorro de energía o la capacidad de producción inmediata o mediata (como ocurriría, por ejemplo, en el caso del valor de una máquina juzgada en cuanto bien de cambio o bien de capital).

d] Lo bueno religioso. Aquello que se aprecia positivamente desde el punto de vista religioso es calificable, in genere, de sagrado. La vivencia de lo sagrado es una vivencia eminentemente afectivo-conativa en la que, por el ministerio de la fe, se vive una comunión con lo absoluto. Consecuentemente, un objeto sólo es religioso en la medida en que entra en una conexión con lo absoluto, en cuanto sirve como lugar de aparición de él, promueve la unión con él o recibe su acción, y, en el caso opuesto, en cuanto obstaculiza la aparición, unión o acción de lo absoluto. De aquí surge la antítesis de lo sagrado y lo profano o impío que concierne a los objetos de la valoración religiosa. Bueno (o malo), religiosamente, es un objeto en conexión con lo absoluto, según que promueva más menos (u obstaculice) la unión del alma con él. Es esta conexión positiva la que se exige aprobar y, eventualmente, fomentar en la va- 
loración positiva y que es comunicada por 'bueno' en su uso religioso.

Decir que $X$ es bueno (desde el punto de vista religioso) $\mathrm{es,}$ por tanto, afirmar que debe tenerse frente $a X$, en cuanto une al absoluto, una actitud positiva.

Se entiende que $\mathrm{X}$ puede representar hechos reales, ob. jetos naturales, entes imaginarios, acciones o personas y que el modo y tipo de relación con lo absoluto variará de acuerdo a las diversas representaciones que de él se tienen en las diferentes religiones o creencias trascendentes. Pero tales variaciones no parecen afectar a la interpretación aquí dada, que posee un carácter suficientemente general.

e] Lo bueno hedonistico y vital. Las vivencias de apreciación son aquí predominantemente afectivo-sensoriales. Corresponden a esa amplia gama de estados y sensaciones que va del placer y el dolor bien localizados en el cuerpo a las vivencias de satisfacción o insatisfacción generales, como las que se dan, por ejemplo, en la euforia o en la desazón. Correspondientemente, el objeto valorado -en su sentido más lato- es, indirectamente, una cosa que provoca agrado o desagrado y, directamente, esas sensaciones y estados mismos. Dicho de otro modo, lo aceptado en la valoración positiva es, en última instancia, una vivencia; sólo por mor de ésta ocurre la adhesión y la busca de las cosas o las personas que la provocan.

Cuando de algo se dice que es bueno, desde el punto de vis. ta hedonístico y vital, se da a entender, pues, que hay que ser favorable a ello en cuanto provoca agrado (o cualquier otra vivencia del mismo estilo) o, más simplemente, que hay que ser favorable a los estados afectivos-sensoriales positivos y a aquello que los causa y promueve. ' $X$ es bueno' significa, por tanto: 'debe tenerse con respecto a $X$ (agradable, cómodo, etc.), una actitud pro.'

Conviene advertir que por más que la interpretación arriba ofrecida ponga énfasis en el papel de los estados afectivos-sensoriales dentro del ámbito axiológico hedonístico y vital, no puede confundirse un enunciado psicológico del 
tipo de 'Juan tiene una vivencia de agrado $\mathrm{X}$ ' con un enunciado valorativo como 'La vivencia $\mathrm{X}$ es buena'. El segundo no supone ninguna afirmación de existencia, a diferencia del primero, y, además, comunica en su núcleo significativo una exigencia de actitud favorable.

f] Lo bueno social y jurídico. Vamos a considerar, como una forma especial de predicado de valor, lo bueno social y jurídico, que es diferente de lo moral en tanto que responde a la relación interindividual basada en lazos de conveniencia, agrado o coacción política. Ciertamente que cabe encontrar muchos puntos en común entre los valores morales y los jurídicos y sociales, pero también es posible y teóricamente fecundo distinguir el punto de vista moral, que implica a la vez internalidad e imperatividad de los principios, del punto de vista de la vida social, con su constricción externa y su exigencia de trato y cooperación.

Los valores de este orden se dan en una vivencia teñida ordinariamente de afectividad; intervienen en ella decisivamente factores intelectuales, bien que, en la mayoría de los casos, las conductas están fuertemente canalizadas por hábitos y automatismos inconscientes. Todas las normas de la prudencia, que pertenecen propiamente a este orden y no al moral, muestran claramente el doble sustento mencionado.

Decir que $X$ es bueno (social y jurídicamente) es hacer una afirmación sobre $\mathrm{X}$ en tanto que está de acuerdo con el orden social, sea en el plano del trato espontáneo de las personas, sea en el de las instituciones legales. Por ejemplo, la puntualidad es socialmente buena en cuanto promueve el acuerdo de los miembros del todo social y conviene a los intereses de todos. Las sanciones penales tienen el mismo signo valorativo por análoga razón.

$\mathrm{Al}$ decir que $\mathrm{X}$ es (social y jurídicamente) bueno se está mencionando pues a X en el contexto de la vida de la comunidad, con sus metas y propósitos, tomados en el sentido más amplio (que incluye satisfacciones compartidas, intereses comunes, vínculos sentimentales, procesos de 
desarrollo y progreso y medidas de prevención y defensa). Respecto a X así entendido se afirma la exigencia de una actitud favorable, según el esquema aplicado en los casos anteriores.

g] Lo bueno teórico. Cuando decimos de un sistema científico, así como de una hipótesis, de un libro o de una concepción, que son buenos, usamos el término 'bueno' en un sentido valorativo específico, el del valor que generalmente se llama teórico o cognoscitivo. La vivencia en que se da este valor - sin ser extraña totalmente a las motivaciones y los elementos afectivo-conativos- es, por antonomasia, la vivencia intelectual. Para tener conciencia del valor teórico de un complejo objetivo es preciso estar en actitud de intelección. Correlativamente, nada que no esté dado como término de una vivencia intelectiva puede ser juzgado teórica o cognoscitivamente bueno.

¿Qué es lo que da su especificidad teórica a la calificación de bueno? Dos cosas principales, en opinión nuestra: la primera es la función del objeto juzgado en el proceso del conocimiento, función que puede, grosso modo, ser de promoción o de obstáculo; la segunda es el tipo de objeto de que se trata, pues las proposiciones son sujetos propios de una enunciación valorativa teórica. Cuando aplicamos 'bueno' a un objeto en cuanto promueve el conocimiento o cuando juzgamos una proposición como tal, la valoración del caso es por lo general teórica.

Según lo anterior, decir que $X$ es (teóricamente o cognoscitivamente) bueno quiere decir que debe tenerse respecto $a$ $X$, como proposición o en cuanto instancia promotora del conocimiento, una actitud favorable $e^{12}$.

Los diferentes sentidos de 'bueno' arriba examinados pueden quizá percibirse mejor en el ejemplo de un objeto

12 Cabe notar que en éste $\mathbf{y}$ otros casos parece haber una instancia originariamente valorada, de la que depende la posibilidad de calificar de bueno a un objeto. En lo que toca al valor teórico, esta instancia sería el conocimiento mismo. La pregunta que queda abierta es: ¿puede decirse del conocimiento que es, él mismo, cognoscitivamente bueno? Si no ¿qué clase de valor es el pertinente? 
valorado según los diferentes órdenes de valor, esto es, según diversas predicaciones de 'bueno' correspondientes a las diferencias examinadas. Sea un trozo de pan; de él se puede decir que es bueno, en sentido estético, considerando su pura apariencia exterior, con prescindencia de todas sus virtualidades o propiedades reales, como es, por ejemplo, la alimenticia. En cambio, se le llamará bueno vital y hedonísticamente en cuanto es capaz de dar salud y producir sensaciones agradables, o sea, justamente por razones que en el caso anterior no eran tomadas en cuenta. Pero también puede hablarse del trozo de pan económicamente; en ese caso, si se lo califica de bueno, será en consideración a su capacidad de servir para la obtención de bienes intercambiables. Por otra parte, un católico podrá valorarlo religiosamente en cuanto el pan promueve la unión del alma con el absoluto, según la creencia en la eucaristía. Es muy difícil, en cambio, que se haga una calificación moral propia del pan en cuanto pan, ya que 'bueno', moralmente entendido, se aplica a acciones y personas. Solamente cabría hablar del valor moral del pan en sentido analógico o traslaticio, como cuando se dice que es un alimento "honesto y humilde".

\section{Observaciones finales}

A manera de recapitulación llamamos la atención sobre los siguientes hechos: a] Según sea la función del objeto y de la vivencia se distinguen tipos diversos del valor que corresponden a sendos significados de la palabra 'bueno'. Estas diferencias vivenciales y objetivas se ofrecen como variaciones de la actitud y de los objetos articuladas con una exigencia de adhesión. b] Las tres clases de variaciones del sentido de 'bueno' que hemos examinado no deben ser tomadas necesariamente como las únicas. No son, además, mutuamente excluyentes; por el contrario, corresponden a clasificaciones diversas del sentido, que obedecen a diferentes principios. c] La unidad y la plurivocidad de 'bueno' 
quedan explicadas sin contradicción por el doble momento esencial del sentido de esta palabra: el momento psicoló. gico y objetivo, variable y múltiple. d] Nada obliga a apelar, como ha sido usual en las teorías axiológicas más difundidas, a la existencia de propiedades o entidades sui generis, eventualmente aprehensibles por vía intuitiva, ni menos a reducir el significado de los enunciados valorativos a meras informaciones o expresiones de hechos vivenciales. e] Ninguna teoría que identifique al valor con una instancia óntica determinada podrá dar cuenta de la multivocidad de la palabra 'bueno'. $f$ ] Ninguna teoría que confunda el valor y el hecho podrá dar cuenta de la trascendencia con respecto a los datos existenciales que es típica en los enunciados de valor y se ofrece como una constante a través de la múltiple variedad de éstos; de allí la necesidad de recurrir a un término normativo como 'debe' (u otros similares) en el análisis del sentido de las oraciones valorativas y en la explicación de su unidad. g] Queda planteada la tarea de interpretar las formas más finas y singures del fenómeno axiológico, echando mano de la misma hipótesis, que sólo así podrá mostrar su entera capacidad explicativa. 
1. The multiple variation of meaning of evaluative words in common evaluating usage is a fact of our linguistic experience. I wish to call attention here to three main classes of variations of 'good':

i] There is what is good or the good of each object with reference to each genus or species of object. It is not the same to speak of a good book as of a good automobile.

ii] One speaks also of good in an absolute sense as opposed to the relatively good, and of intrinsic as oppossed to extrinsic good, and likewise of good as an end and as a means, as primary and derived, etc. There is also what is good in a comparative sense, and the degrees of good.

iii] Thirdly, there is what is good according to the orders of the esthetic, the moral, the economic, the theoretical, etc.

Does not this multiplicity of meaning conspire against the semantic unity of good? It is not a matter of an ordinary case of a homonym, of unusual usage, of alteration or loss of meaning. Far from affecting the unity of its use and function, its multivocal cha. racter seems to constitute an essential feature of the meaning of 'good', and to open the way to the understanding of its nature.

In a first approximation, 'good' has the character of a formal term, which means that, as such, it does not communicate any ontic content but can adapt itself to diverse contents and, with them, specify their signification. It qualifies the respective objective com. plexes, without its meaning becoming fixed by this commerce with such contents.

The fact that stands out is that the unity of the semantic behavior of the term 'good' is given by lived experiences which manifest the same essential structure in all cases. Those who pronounce or hear the word 'good' behave in basically the same manner with respect to the object, that is, with an attitude that is favorable or pro.

In what follows, the connection between the invariable aspect and the variations of the meaning of 'good' is examined from a general point of view. As a semantic hypothesis, the following is proposed: 'good' communicates the demand of a favorable attitude, referred to an element or a determined objective complex, that is incorporated in the meaning of the word in each particular case. This demand bears within it a moment of universal and unconditional imperative. ness of a nonfactical order that remains unchanged in all cases, giving to "good" a unitary meaning. Since the common element which 
is communicated is not an ontic content but a demand, it is impossible to know once and for all what it is that is demanded and to what object or characteristic we ought to hold.

On the other hand, the plurality is explained by the variation of the elements or objective complexes that are estimated in each case, and by the character and the degree of the favorable attitudes, these being variable in accordance with the wide range of human behaviour.

On a second level of analysis, and taking into account the three factors of the evaluative phenomenon that are reflected in the semantic hypothesis proposed here, namely: the object, the attitude or lived experience, and the demand, in what follows an attempt is made to explain the differences corresponding to each type and case of variation.

2. With respect to the value proper to each genus of object, the relation of difference that there is in 'good' between the descriptive content and the properly evaluative moment is pronounced. 'Good' en each case has to do with certain properties of the estimated object, but in using this term we do not simply describe these properties, for they rather figure as signs of a special condition of the object, the condition of possessing value.

As support for this thesis, reference is made, in the first place, to the impossibility in the language of value of reducing the evaluative meaning to the descriptive content; and, in the second place, to the difference between the learning of 'good' and that of other words like 'blue', 'left'.

To sum up, in each usage of "good" applied to each species of things, there is a variable element, namely, that corresponding to the characteristics of that object; and there is a constant element, the demand of a favorable attitude with respect to the mentioned objective complex.

3. With respect to the difference between absolute and relative value and the rest of the variations mentioned in (ii), it may be observed that when one formulates a judgment of absolute or intrinsic value, one takes into account only the intrinsic properties of the object. In the translation of 'good' according to the hypothesis, in saying that something is intrinsically good, one communicates that there is an unconditional demand to adopt an atittude pro or favorable to the object, to the exclusion of other injunctions that may be present.

In ordinary sentences that express relative value, the objective semantic nucleus is constituted by terms of the type 'good for' or 
'good in relation with'. The main case is that of the relation of means to end; in this case an object is valued in so far as it is presented as an injunction directed to the realization of an end. Nevertheless, the analysis in terms of demand does not have to be substantially changed. The demand of ought continues to be as full and unconditional as in the case of the intrinsically good, although the object itself is not considered unconditionally.

Examining next comparative evaluative judgements, it may be noted that in all these there is, as a semantic nucleus, an intentional ingredient of 'good in relation to', which brings this case close to the preceding one.

With respect to statements that formulate an evaluative judgment of degree, two possible interpretations are studied: a] what varies is the demand - or the ought; b] what varies is the attitude or lived experience. The second alternative is adopted, in view that the first threatens to psychologize the evaluative demand. On the other hand, variation can be perfectly acceptable in the case of the attitude, that is, of a lived experience. According to this, to say that $\mathrm{X}$ is best would amount to saying that one must have with respect to $\mathrm{X}$ the maximum degree of propensity or the most favorable attitude.

4. Next are studied the variations of 'good' corresponding to the following orders of value: the esthetic, moral, economic, religious, vital-hedonistic, socio-juridical and theoretical. In each of these cases, the evaluative differentiation is explained, whether by reference to the object or to the type of lived experience or to both, in such a way that the formula $X$ is good will mean something distinct depending upon how $\mathrm{X}$ is understood and upon the lived experience that is demanded. Very briefly, the differences indicated are: a] $\mathrm{X}$ as a purely apparent or unreal injunction, given in an imaginative lived experience, characterizes the esthetic usage of 'good'; b] X corresponding to actions of the will and to a demand of deliberately realizable actions is what is characteristic of the moral usage of 'good'; c] the ability of $\mathrm{X}$ to serve for the obtaining of the maximum of products with the minimum of effort, the determination of which requires a calculation of real possibilities and effects, is what differentiates the economic meaning of 'good'. d] the vinculum with the absolute and the promotion of this vinculum, usually supported in an affective-conative lived experience, is what is central in the religious meaning of 'good'; e] the vital-hedonistic good bears within it a determination of $\mathrm{X}$ as agreeable or inducing of satisfaction; $\mathrm{f}]$ the socio-juridical good is linked with the function of $\mathrm{X}$ as a promoter of interindividual connections and as adequate for the conveniences 
and interests of the group; g] finally, the theoretical or cognitive usage of 'good' rests on the positive function of $\mathrm{X}$ in knowledge.

5. By way of recapitulation, attention is called to the following points: a] Depending on the function of the object and of the lived experience, diverse types of value that correspond to the respective meanings of the word 'good' are distinguished. b] The three classes of variations of the meaning of 'good' ought not necessarily be taken as the only ones. c] The unity and multivocal character of 'good' are explained without contradiction by the essential double moment of the meaning of this word: the unitary nonfactical moment of the demand, and the psychological, subjective, variable and multiple moment. d] Nothing obliges us to appeal to the existence of sui generis properties or entities nor to reduce evaluative statements merely to information about or expressions of facts of lived experiences. e] No theory that identifies value with a determined ontic injunction would be able to take account of the multivocal character of the word 'good'. f] No theory that embraces both value and fact would be able to take account of the transcendence with respect to existential data that is typical of statements of value; hence the necessity of having recourse to a normative term like 'ought' in the analysis of the meaning of evaluative sentences and in the explana. tion of their unity. g] The hypothesis proposed here ought to be tested in the analysis of more refined variations.

[Traducción de J. Schönberg] 